\title{
Bioplastiche: sono realmente così innocue?
}

\author{
Cristina Mastrolia ${ }^{1}$
}

${ }^{1}$ Affiliation not available

\begin{abstract}
Le bioplastiche, fin dal loro inserimento nei processi produttivi, sono state considerate come una alternativa sicura ed ecosostenibile alle "normali" plastiche (a base di petrolio) ree di danneggiare invariabilmente l'ecosistema, in particolare quello marino. Di fatti, è stimato che circa il 56\% della plastica prodotta annualmente non venga riciclata o incenerita, bensì venga scartata soprattutto negli oceani in forma di microplastiche. Di conseguenza, la ricerca sulle bioplastiche e specificamente sul loro ciclo vitale è un campo di studio di notevole interesse ed in rapida crescita. Tuttavia, pensare alle bioplastiche come la perfetta soluzione al problema dell'inquinamento da plastica sarebbe un grossolano errore nonostante la loro produzione avvenga a partire da fonti rinnovabili. Infatti, la maggior parte delle bioplastiche sono biodegradabili ma non compostabili.

Il fine di questo lavoro è quindi quello di dare luce alla parte meno conosciuta, e spesso ignorata, delle bioplastiche; ovvero il loro potenziale dannoso sia durante la loro produzione, sia nei vari processi di smaltimento, ed in particolare in ambiente marino; nonché sull'applicazione del criterio e delle tecnologie di economia circolare per il recupero, ed il riutilizzo delle bioplastiche all'interno degli impianti di smaltimento delle acque reflue.
\end{abstract}

\section{Le bioplastiche e la loro produzione}

La produzione a partire da risorse rinnovabili, sia da biomasse che da biopolimeri, ha rappresentato da sempre la maggiore attrattiva delle Bioplastiche (BPLs); soprattutto per la notevole variabilità 
della natura di queste risorse, e di conseguenza nella mutevolezza degli utilizzi che si possono ricavare dalle diverse BPLs.

Le Bioplastiche vengono distinte in cinque famiglie in base al loro materiale d'origine:: a base di Amido, a base di Cellulosa, a base Proteica, a base Alifatica ed a base organica ${ }^{1}$.

Tuttavia, nonostante gli innegabili aspetti positivi e miglioramenti rispetto alle classiche plastiche a base di petrolio, considerate le principali responsabili dell'inquinamento con una stima di 269,000 tonnellate di plastica (Eriksen, 2014) che attualmente navigano gli oceani, bisogna ricordare che, malgrado la loro origine a partire da fonti rinnovabili, e la loro decomposizione principalmente in acqua ed anidride carbonica, non tutta la bioplastica riesce ad essere completamente smaltita senza danni all'ambiente ${ }^{2}$. Ed è per questo che la classificazione più utilizzata si basa sulla biodegradabilità delle stesse . Le plastiche, in generale, possono essere di origine biologica o fossile, entrambe biodegradabili e non biodegradabili; in particolare, le plastiche non biodegradabili di origine fossile sono le plastiche convenzionali (da petrolio) mentre le altre sono bioplastiche.

Per quel che concerne invece il mercato, a livello globale la bioplastica più utilizzata è la PLA (47\%); al secondo posto quelle a base di amido (41\%) (European Bioplastics).

Le aree di produzione in cui vi è una maggiore applicazione delle BPLs sono ${ }^{1}$ :

- Imballaggio: utilizzate specialmente per imballaggi di cibi e confezioni d'asporto. Vengono preferite le BPLs a base di amido e PLA per la facilità di produzione.

- Dispositivi medici: utilizzate specialmente per fili di sutura, protesi ed impianti. Il 95\% dei dispositivi medici a base di BPLs è rappresentato da Fili per Sutura in PLA.

- Agricoltura: l'applicazione delle BPLs in questo campo è in costante crescita, in quanto rappresentano le applicazioni più promettenti. Vengono utilizzate, sotto forma di pellicole per serre, reti e buste per fertilizzanti.

- Altre Applicazioni: utilizzo più di nicchia è rappresentato dall'industria elettronica ed auto- 
mobilistica. La BPL più utilizzata è il PLA grazie alla sua stabilità e possibilità di una precisa lavorazione durante i processi industriali.

\section{Biodegradabilità delle Bioplastiche}

La biodegradazione è un processo naturale basato sulla completa mineralizzazione di materiali in anidride carbonica, acqua, ammonio, nitrogeno e biomassa. Questo processo, influenzato da UV, ossigeno e calore avviene grazie a microrganismi, quali batterie alghe e funghi, che frammentano i materiali in piccole molecole e lo ossidano ${ }^{3}$.

I materiali biodegradabili sono ad oggi preferiti, in quanto ciò ne permette lo smaltimento non tossico da parte di microrganismi durante i loro processi vitali. Il processo di biodegradazione segue tre fasi comuni ${ }^{1}$ :

- Bio-deterorazione: modifiche delle proprietà fisiche, chimiche e meccaniche dei polimeri per la crescita di microrganismi al loro interno o sulla loro superficie.

- Bio-frammentazione: degradazione in oligomeri o monomeri dei polimeri.

- Assimilazione: frammentazione di oligomeri e monomeri da parte di microrganismi, con il rilascio di atomi di carbonio, che porta alla formazione di anidride carbonica, acqua e biomassa.

Per quanto concerne la stima del grado di biodegradabiltà delle BPLs, vengono comunemente utilizzati quattro metodi ${ }^{3}$ :

1. Produzione di $\mathrm{CO} 2 \mathrm{e} / \mathrm{o} \mathrm{CH} 4$ durante il processo di biodegradazione.

2. Monitoraggio della riduzione del peso delle bioplastiche nel tempo.

3. Morfologia della superficie e Peso molecolare dei materiali. 
4. Misurazione della Domanda Biologica di Ossigeno (BOD) o rapporto tra BOD e Domanda Teorica di Ossigeno (BOD/ThOD).

Favorevole caratteristica delle BPLs è la possibilità di venir degradate sia in ambiente aerobico che in ambiente anaerobico. In particolare, il processo di biodegradazione aerobica viene monitorato tramite lo studio dell'Indice di assimilazione microbica e l'Indice di biodegradazione (ovvero perdita di massa). Quest'ultima dipende dalla temperatura utilizzata dal processo; è stato riscontrato una percentuale di biodegradazione dimezzata a temperature di $35-37^{\circ} \mathrm{C}$ rispetto a $\mathrm{T}$ più alte $\left(55^{\circ} \mathrm{C}\right)$. Uno studio di Cho et al., ha riscontrato una biodegradazione di BPL dell' $88 \%$ in 44 giorni. Diversamente, per la biodegradazione anerobica, non presenta gli stessi risultati per tutte le bioplastiche ${ }^{3}$, infatti, sono stati ottenuti valori di degradazione dal 60 al $92 \%{ }^{4}$.

A prescindere dal metodo di monitoraggio e dalla presenza o meno di ossigeno, gli ambienti favorevoli per la biodegradazione sono il suolo ed il compost. In particolare, biodegradazione in compost può essere effettuata sia in scala domestica che industriale, malgrado sia necessario, per normativa, una disintegrazione di almeno il 90\% del materiale in frammenti più piccoli di 2 mm e una perdita di almeno $90 \%$ del peso in un tempo di sei mesi.

\section{Ecologicità delle Bioplastiche}

Spesso, i concetti di "biodegradabilità" e di "ecologico" vengono utilizzati come sinonimi. Tuttavia, la realtà è profondamente diversa. Per essere "ecologico" un materiale non deve avere impatti negativi sull'ambiente: di fatti, anche una completa biodegradabilità nell'ambiente non indica ne- 
cessariamente la mancanza di un impatto negativo, seppur minimo. E' questo il caso delle bioplastiche. Per identificare l'entità degli impatti ambientali causati dalle BPLs, è stato improntato un metodo volto alla Valutazione del Ciclo di Vita (LCA) di questi nuovi materiali, seguendo un approccio "cradle-to-grave" (dalla culla alla tomba), ovvero dalla produzione fino allo smaltimento. Gli impatti ambientali considerati sono ${ }^{5,6}$ :

- Potenziale di Riscaldamento Globale (GWP): rilascio di gas serra.

- Potenziale di Acidificazione (AP): rilascio di composti a potenziale acido.

- Potenziale di Eutrofizzazione (EP): aumento della biomassa per eccessiva fertilizzazione di terreno ed acque.

- Potenziale Tossico (TP): rilascio di sostanze tossiche in acqua, aria ed ambiente.

- Potenziale di Esaurimento dei Fossili (FDP): consumo di materiale fossile.

Partendo dal processo di produzione, secondo l'approccio cradle-to-grave, impatti negativi si riscontrano già durante la coltivazione e la raccolta delle materie prime. Di fatti, la richiesta -in costante crescita- di BPLs, causa un'aumentata pressione sui raccolti e, di conseguenza, deforestazione. Per velocizzare la crescita delle materie prime, inoltre, queste vengono geneticamente modificate con rischi ambientali; infatti la produzione di questa biomassa provoca Eutrofizzazione e rilascio di sostanze tossiche ${ }^{7}$.

Per quello che riguarda invece la manifattura delle BPLs, i processi utilizzano, al giorno d'oggi, energia non rinnovabile (NREU) ${ }^{8}$. Ciò implica il rilascio di Gas Serra che aumentano il Potenziale di Riscaldamento Globale, oltre al rilascio di mercurio e $\mathrm{SO}_{2}$ che causano problemi respiratori. Per quanto concerne l'impatto su suolo ed aria, si ha un alto Potenziale Tossico causato dalle sostanze rilasciate da NREU in quanto causa di piogge acide che inquinano terreno e ambiente marino ${ }^{8,9}$. 


\section{Comportamento delle bioplastiche in ambiente acquatico}

Nonostante il fatto che idealmente, come già discusso, il ciclo vitale delle bioplastiche debba terminare con il compostaggio delle stesse, a causa delle limitazioni dei sistemi di rimozione degli inquinanti (WWTPs) $)^{10,11,12}$ e della disseminazione di rifiuti, una parte delle bioplastiche raggiunge l'ambiente marino ${ }^{13}$. Per quanto concerne la loro degradazione, fino ad ora non è stata osservata possibilità di biodegradabilità in acqua, tuttavia è stata riscontrata una frammentazione di BPLs in Bio-Microplastiche (BMPs) ${ }^{14}$.

Il tasso di degradazione dei rifiuti bioplastici in ambiente acquatico è stato studiato da M. Tosin et al. ${ }^{15}$ in cui sono state simulate in laboratorio tre zone marine (pelagica, eulittorale e sublitorale), rispettivamente in un acquario contenente acqua marina, sull'interfaccia sabbia-acqua e, infine, simulando l'affondamento di un rifiuto in acqua.

In particolare:

- Zona Pelagica: I campioni sono stati rispettivamente estratti dopo 3, 8, 12, e 19 mesi riscontrando un decadimento delle proprietà meccaniche dal $66 \%$ al $96 \%$.

- (b) Zona Eulittorale: . Dopo 9 mesi, solo pochi frammenti erano distinguibili.

- (c) Zona Sublitorale: è stato valutato il BOD, risultando in un consumo totale di $\mathrm{O}_{2}$ di circa $120 \mathrm{mg} / \mathrm{L}$. In particolare, dopo 236 giorni, è stata riscontrata una biodegradazione media dell' $88,2 \%$

I risultati di questa ricerca hanno dimostrato che nella zona eulittorale, grazie alla maggiore esposizione alla luce solare e ai sedimenti che sommergono le bioplastiche, vi è una più completa degradazione. Ulteriori studi per la valutazione di come differenti temperature, pressioni, flussi marini e luce e rapporti con i sedimenti modifichino la biodegradazione delle BPLs si rendono quindi 
fondamentali per una comprensione completa, a tutto tondo, di come le bioplastiche interagiscono con l'ambiente marino.

La presenza di queste bioplastiche mal degradate, purtroppo, non porta solo un danno estetico alle acque dei mari e degli oceani, ma i frammenti di Bio-Microplastiche si inseriscono all'interno dei cicli vitali degli animali marini, in particolare degli invertebrati: con conseguenze, come si può immaginare, sia sugli stessi che sui loro predatori, nonché sull'uomo ${ }^{14}$.

\section{Ruolo degli impianti di trattamento delle acque reflue}

Gli impianti di trattamento delle acque reflue (WWTPs) rappresentano uno dei maggiori affluenti di microplastiche nelle acque ${ }^{10,16,17,18}$. Inoltre, i processi di depurazione delle acque stanno sollevando dubbi ${ }^{19}$ a causa delle emissioni di gas serra, dei costi elevati di manutenzione e spreco di risorse rinnovabili ${ }^{20,21,22}$. Ai fini di limitare tali effetti negativi, è stato valutato un loro ruolo nell' economia circolare $^{23}$, in cui è incoraggiata una trasformazione degli impianti in Strutture di Recupero ${ }^{24,25,26}$. Una delle possibili metamorfosi, è quella di convertire gli WWTPs in impianti di produzione di PHAs: invece di convertire materia organica in anidride carbonica, la biomassa potrebbe essere utilizzata come polimero per la produzione di bioplastica. In uno studio svolto sull'impianto di Wetterskip Fryslan, è stato riscontrato un ritorno economico dalla produzione di PHA di circa 20/50 volte maggiore rispetto alla corrente produzione di biogas. Inoltre, la produzione di PHA dai fanghi attivi ${ }^{27}$ dei WWTPs comporta anche una riduzione dell'impronta di carbonio dell'impianto di circa il $21 \%{ }^{28,29}$.

Il motivo per cui tale conversione non è ancora stata effettuata, risiede nel fatto che per raggiungere tale percentuale di riduzione dell'impronta di carbonio è necessario un impianto di notevoli 
dimensioni, e di conseguenza notevoli costi. In più, il prodotto risultante da questo processo sarà di scarsa qualità, andando a restringere il già povero mercato delle BPLs.

Un ulteriore sfruttamento degli WWTPs può essere effettuato con l'utilizzo delle microalghe presenti nell'impianto come organismi producenti bioplastiche. Le microalghe, a differenza delle altre piante utilizzate per la produzione di BPLs, non necessitano di acqua potabile e suolo per crescere, riducendo di conseguenza drasticamente i costi delle materie prime e della raccolta. Fattore vantaggioso è anche il tempo di crescita in quanto, nelle giuste condizioni ambientali, sono in grado di duplicare la loro biomassa in poche ore ${ }^{30}$.

\section{Smaltimento delle Bioplastiche}

Proseguendo con il nostro approccio cradle-to-grave per la valutazione del Ciclo di Vita delle bioplastiche, prenderemo ora in considerazione diverse opzioni di smaltimento.

I vantaggi e gli svantaggi dei vari metodi analizzati ${ }^{31}$, sono riportati in Fig.1.

Oltre alle problematiche legate ai metodi di smaltimento ${ }^{32}$, è motivo di preoccupazione la confusione del termine biodegradabile con eco-sostenibile, che causa un aumento di rifiuti gettati nell'ambiente, andando ad aumentare il già elevato inquinamento da plastica ${ }^{33}$. Inoltre, la mancanza di una corretta etichettatura delle bioplastiche e una raccolta non differenziata dalle normali plastiche causa una difficoltà nel recupero di tali materiali che, sommato agli elevati costi di smaltimento, non incentiva il trattamento di rifiuti di bioplastica da parte dei gestori di impianti di smaltimento. 


\section{Possibili Soluzioni}

Le problematiche fin ad ora esposte non possono essere facilmente risolte in tempi brevi. Il primo passo sarebbe quello di istruire il pubblico generale tramite campagne di sensibilizzazione. In aggiunta, dovrebbero essere aggiornate le norme governative a riguardo di etichettatura e differenziazione dei rifiuti secondo polimero di origine.

Oltretutto si rende necessario lo sviluppo di tecnologie avanzate che utilizzino energie rinnovabili e minore energia per lo smaltimento di tali prodotti. Infine, per incentivare l'utilizzo delle bioplastiche, potrebbero essere avviati programmi di fondi o riduzioni sui costi e sulle tasse per la produzione e lo smaltimento delle bioplastiche ${ }^{31,34}$.

\section{Conclusioni}

Lo scopo di questo articolo è quello di dar luce alle problematiche, spesso misconosciute o ignorate, delle bioplastiche. Anche se esse presentano numerose caratteristiche positive rispetto alla plastica a base di petrolio, tramite l'approccio Cradle-to-Grave è evidente come le BPLs abbiano impatti anche negativi sull'inquinamento. A partire dalla raccolta delle materie prime e della produzione, è stato valutato l'impatto negativo delle BPLs su suolo, aria ed acqua a causa di coltivazioni intensive ed abuso di sostanze chimiche durante la coltivazione. Gli attuali impianti di produzione, a causa dell'utilizzo di energia non rinnovabile contribuiscono al riscaldamento globale, acidificazione, eutrofizzazione e tossicità nell'ambiente. Oltre a quanto già detto, ruolo di spicco è occupato dagli impianti di trattamento delle acque reflue che, pure essendo una delle cause dell'inquinamento acquatico, possono avere un importante ruolo in un ciclo produttivo del- 
le bioplastiche più sostenibile. Per quanto concerne invece lo smaltimento delle bioplastiche, è stato evidenziato come la cattiva gestione dei rifiuti, imputabile a molteplici fattori, ha incrementato l'inquinamento da plastiche in ambiente marino. L'articolo ha discusso la degradazione di Bio-Microplastiche all'interno di zone acquatiche (pelagica, eulitorale e sublitorale) riscontrando una più efficace degradazione a livello dell'interfaccia acqua-sabbia rispetto alla degradazione in sola acqua. Infine sono stati valutati i vantaggi e gli svantaggi delle diverse tecnologie di smaltimento, evidenziando falle sia nell'organizzazione politica che mancanze delle esistenti tecnologie, manifestando la necessità di tecnologie più avanzate e sostenibili e il bisogno di campagne di sensibilizzazione per educare sul vero significato di Biodegradabilità e Sostenibilità, nonché sulle caratteristiche delle Bioplastiche.

\section{References}

1.S.A., A. Introduction to Bioplastics Engineering. (Elsevier, 2016).

2.Singh, P. Bio-Plastics: A sustainable alternative to conventional petroleum based plastics. International Journal of Advanced Scientific Research and Management 4, (2019).

3.Folino, A., Karageorgiou, A., Calabrò, P. S. \& Komilis, D. Biodegradation of Wasted Bioplastics in Natural and Industrial Environments: A Review. Sustainability 12, 6030 (2020).

4.Bátori, V., Åkesson, D., Zamani, A., Taherzadeh, M. J. \& Horváth, I. S. Anaerobic degradation of bioplastics: A review. Waste Management 80, 406-413 (2018).

5.Changwichan, K., Silalertruksa, T. \& Gheewala, S. Eco-Efficiency Assessment of Bioplastics Production Systems and End-of-Life Options. Sustainability 10, 952 (2018).

6.Azapacig. Polymers: the environmental and sustainable development. (2003).

7.Pathak. Bioplastics: Its Timelined Based Scenario \& Challenges. Journal of Polymer and 
Biopolymer Physics Chemistry 2 (4), (2014).

8. Yates, M. R. \& Barlow, C. Y. Life cycle assessments of biodegradable commercial biopolymers-A critical review. Resources, Conservation and Recycling 78, 54-66 (2013).

9.How Does Non Renewable Energy Affect The Environment? - Green Tech Talk.

10.Naddeo, V. et al.. Removal of contaminants of emerging concern from real wastewater by an innovative hybrid membrane process - UltraSound Adsorption and Membrane ultrafiltration (USAMe®). Ultrasonics Sonochemistry 68, 105237 (2020).

11.Borea, L. et al.. Wastewater treatment by membrane ultrafiltration enhanced with ultrasound: Effect of membrane flux and ultrasonic frequency. Ultrasonics 83, 42-47 (2018).

12.JJ Lado; V Cartolano, V. N. Performance analysis of a capacitive deionization stack for brackish water desalination. Desalination 501, (2021).

13.Preparation of novel polyvinylidene fluoride (PVDF)-Tin(IV) oxide (SnO2) ion exchange mixed matrix membranes for the removal of heavy metals from aqueous solutions. Separation and Purification Technology 250, (2020).

14.Anderson, G. \& Shenkar, N. Potential effects of biodegradable single-use items in the sea: Polylactic acid (PLA) and solitary ascidians. Environmental Pollution 268, 115364 (2021).

15.Tosin, M., Weber, M., Siotto, M., Lott, C. \& Innocenti, F. D. Laboratory Test Methods to Determine the Degradation of Plastics in Marine Environmental Conditions. Frontiers in Microbiology 3, (2012).

16.Pervez, M. N. et al. . A critical review on nanomaterials membrane bioreactor (NMs-MBR) for wastewater treatment. npj Clean Water 3, (2020).

17.Self-forming Dynamic Membranes for Wastewater Treatment.

18.Fraiese, A. et al.. Ultrasonic processes for the advanced remediation of contaminated sediments. 
Ultrasonics Sonochemistry 67, 105171 (2020).

19.Ibrahim, Y., Abdulkarem, E., Naddeo, V., Banat, F. \& Hasan, S. W. Synthesis of super hydrophilic cellulose-alpha zirconium phosphate ion exchange membrane via surface coating for the removal of heavy metals from wastewater. Science of The Total Environment 690, 167-180 (2019).

20.Ali, A. A. A., Naddeo, V., Hasan, S. W. \& Yousef, A. F. Correlation between bacterial community structure and performance efficiency of a full-scale wastewater treatment plant. Journal of Water Process Engineering 37, 101472 (2020).

21.Naddeo, V., Landi, M., Scannapieco, D. \& Belgiorno, V. Sonochemical degradation of twenty-three emerging contaminants in urban wastewater. Desalination and Water Treatment 51, 6601-6608 (2013).

22.Fortunato, L., Ranieri, L., Naddeo, V. \& Leiknes, T. O. Fouling control in a gravity-driven membrane (GDM) bioreactor treating primary wastewater by using relaxation and/or air scouring. Journal of Membrane Science 610, 118261 (2020).

23.Taherzadeh, V. N. M. J. Biomass valorization and bioenergy in the blue circular economy. Biomass and Bioenergy 149,

24.Abdallah, H. et al.. Amino-functionalized mesoporous nano-silica/polyvinylidene fluoride composite as efficient ultrafiltration membrane. DESALINATION AND WATER TREATMENT 205, $63-75$ (2020).

25.Jallouli, S. et al.. Efficient and sustainable treatment of tannery wastewater by a sequential electrocoagulation-UV photolytic process. Journal of Water Process Engineering 38, 101642 (2020).

26.Abdulkarem, E., Ibrahim, Y., Naddeo, V., Banat, F. \& Hasan, S. W. Development of Polyethersulfone/-Zirconium phosphate (PES/-ZrP) flat-sheet nanocomposite ultrafiltration mem- 
branes. Chemical Engineering Research and Design 161, 206-217 (2020).

27.ElNaker, N. A., Elektorowicz, M., Naddeo, V., Hasan, S. W. \& Yousef, A. F. Assessment of Microbial Community Structure and Function in Serially Passaged Wastewater Electro-Bioreactor Sludge: An Approach to Enhance Sludge Settleability. Scientific Reports 8, (2018).

28.Ajao. Circularity in wastewater treatment plants :drivers and barriers to the commercialisation of bioplastics from wastewater. (2020).

29.Corpuz, M. V. A. et al. Wastewater treatment and fouling control in an electro algae-activated sludge membrane bioreactor. Science of The Total Environment 786, 147475 (2021).

30.Rocha, C. J. L. et al. Development of bioplastics from a microalgae consortium from wastewater. Journal of Environmental Management 263, 110353 (2020).

31.Ren, X. Biodegradable plastics: a solution or a challenge?. Journal of Cleaner Production 11, (2003).

32.Naddeo, V. \& Korshin, G. Water energy and waste: The great European deal for the environment. Science of The Total Environment 764, 142911 (2021).

33.Naddeo, V. Development of environmental biotechnology and control of emerging biological contaminants: the grand challenge for a sustainable future. Water Environment Research 92 , 1246-1248 (2020).

34.Maheshwari. Eco-Friendly Bioplastic for Uncontaminated Environment. Research Journal of Chemical and Environmental Sciences 1, (2013).

35.Errami, M. \& Garner, H. A tale of two citations. Nature 451, 397-399 (2008).

36.El-malek, F. A., Khairy, H., Farag, A. \& Omar, S. The sustainability of microbial bioplastics production and applications. International Journal of Biological Macromolecules 157, 319-328 (2020). 
37.Siracusa, V., Rocculi, P., Romani, S. \& Rosa, M. D. Biodegradable polymers for food packaging: a review. Trends in Food Science \& Technology 19, 634-643 (2008).

38.Naddeo, V. B., Luigi Rizzo. Water, wastewater and soil treatment by advanced oxidation processes (AOPs). (Edizioni ASTER, 2011). 
Figure Captions

Figure 1. Fig.1 Vantaggi e Svantaggi degli attuali metodi di smaltimento ${ }^{31}$ 
Figures

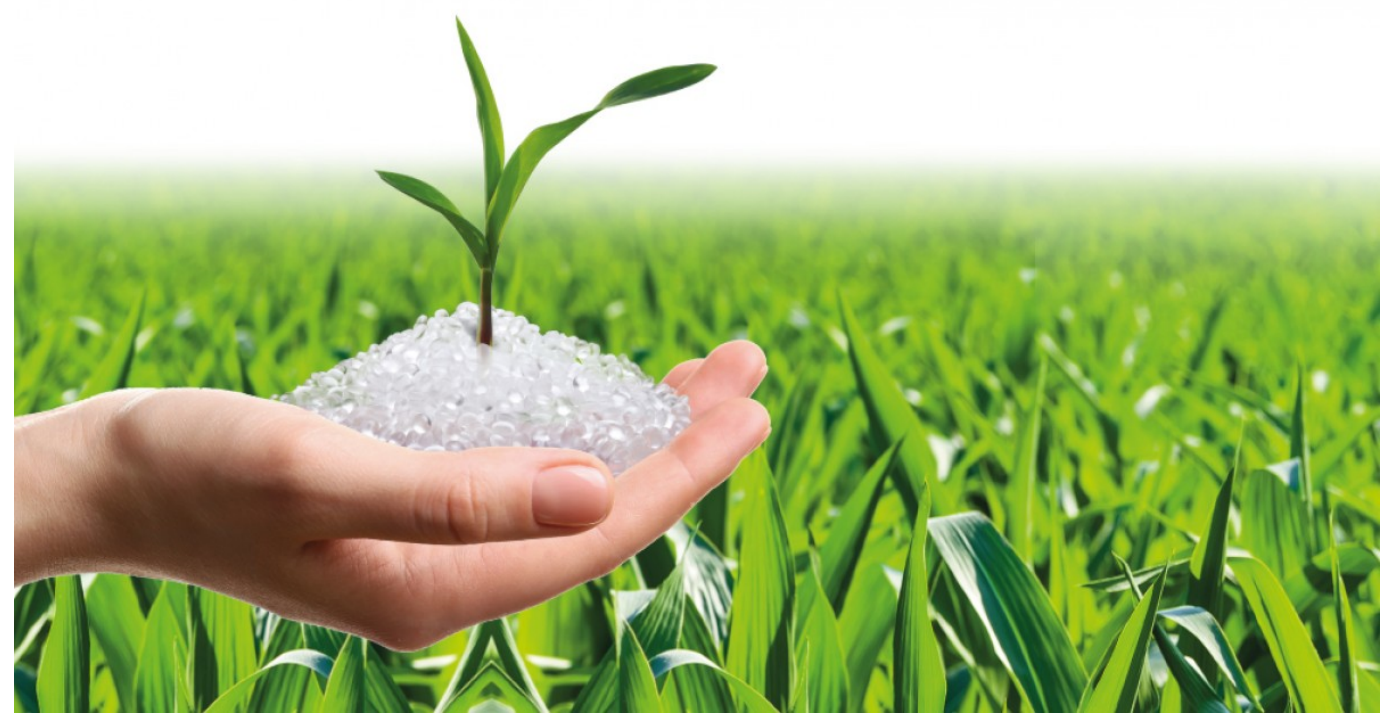




\begin{tabular}{|c|c|c|}
\hline Metodo & Vantaggi & Svantaggi \\
\hline Riciclaggio & $\begin{array}{l}\text {-riduce accumulo di } \\
\text { rifiuti } \\
\text { - risparmia risorse ed } \\
\text { energia per produrre } \\
\text { nuovo materiale } \\
\text { - aumenta la vita dei } \\
\text { prodotti }\end{array}$ & $\begin{array}{l}\text { - alti costi } \\
\text { - consumo di energia } \\
\text { - emissione di } \\
\text { inquinanti } \\
\text { - bassa qualità dei } \\
\text { prodotti }\end{array}$ \\
\hline Compostaggio & $\begin{array}{l}\text {-riduce carico su } \\
\text { discariche } \\
\text { - prodotto finale } \\
\text { utilizzabile nel terreno } \\
\text { - richiede meno } \\
\text { energia }\end{array}$ & $\begin{array}{l}\text { - alti costi } \\
\text { - alte prestazioni e } \\
\text { condizioni richeste } \\
\text { - poco mercato per } \\
\text { compost } \\
\text { - bassa qualità del } \\
\text { prodotto }\end{array}$ \\
\hline Discarica & $\begin{array}{l}\text {-luogo finale per rifiuti } \\
\text { - facile da costruire ed } \\
\text { utilizzare }\end{array}$ & $\begin{array}{l}\text { - mancanza di siti } \\
\text { - alti costi } \\
\text { - emissione di gas } \\
\text { - rilascio di inquinanti }\end{array}$ \\
\hline
\end{tabular}

Figure 1: $\quad$ Fig.1 Vantaggi e Svantaggi degli attuali metodi di smaltimento ${ }^{31}$ 